\title{
Conceiving a Vision within Artificial Intelligence Environments
}

\author{
Prof Dr M S S El Namaki, \\ Victoria University, Switzerland
}

\begin{abstract}
Vision is a depiction of a future state an organization, a corporate culture, a technology or an activity may aspire to realise, and the relative position of the organization within that time-space continuum. It is a conceptualisation of a new and desirable future reality that can induce achievement and motivate followers. It is the business leaders who conceive visions and leading business corporations towards fulfilling them. This article has it focus on identifying types of vision and the building stones to reach them.

The article starts with the definitions of the two objective variables: Vision and Artificial Intelligence. It then explores the likely impact of Artificial Intelligence and Data Sciences on the prospective visions of tomorrow. And it concludes with a framework for vision conception under the emerging driving forces of that no so distant future.

The outcome of this work would be useful in guiding the process of strategic management of businesses under the emerging disruptive conditions of the Artificial Intelligence and Data Sciences.
\end{abstract}

Keywords: Artificial Intelligence; strategic management; vision; Data Analytics 


\section{Introduction}

Vision is a description of something in the future: an organization, a corporate culture, a business, a technology or an activity, and the relative position of the organization within that space. Conceiving a vision has never been as critical as today. The era of data sciences and artificial intelligence is upon us and the disruptive implication of those forces calls for a significant shift in the content of visions and the pertinent visionary leadership (El Namaki, 2017). What type of vision and how would it be conceived will be the focus of the following article.

\section{Basic contours of Artificial Intelligence}

Oxford Dictionary defines artificial intelligence as "the theory and development of computer systems able to perform tasks normally requiring human intelligence, such as visual perception, speech recognition, decision-making, and translation between language". Put differently, AI is the simulation of human intelligence processes by machines, especially computer systems. These processes include learning (the acquisition of information and rules for the use of this information), reasoning (using rules to reach approximate or definite conclusions) and self-correction (self-initiated adjustment or mending of errors and malfunctions). AI relates to many sciences from computing, mathematics and data to psychology, philosophy and linguistics.

\subsection{Present day contours of Artificial Intelligence:}

These present day intelligent systems are able to handle massive volumes of data but lack the analytical and independent self-awareness element that will be the key to building future intelligence. They are either reactive or limited memory.

- Reactive. These are equipment that analyzes possible moves, their own and their opponent's, and choose the most strategic move. They do not have the ability either to form memories or to use past experiences in order to guide current decisions. The computer's perception of the world is direct and it acts according to what it sees.

○ Limited memory (corrective). These equipment use past experience in order to influence future decisions. They can look into the past but past information are only transient and are not saved as part of a library or a learning experience (Hintze, 2016).

\subsection{Future event Artificial Intelligence:}

Systems within this AI segment do not only form representations about its own world, but also about other agents or entities in the world. They do not only understand consciousness, but have it.

- Theory of mind. This is a psychology term. It refers to the understanding that others have beliefs, desires and intentions that impact upon the decisions that they make (Friedlander and Franklin, 2008). Also those others have beliefs, desires, intentions, and perspectives that are different from one's own. This kind of AI does not yet exist.

International Journal of Management and Applied Research, Vol. 6, No. 1 
- Self-awareness. In this category, AI systems have a sense of self and consciousness. Machines with self-awareness understand their current state and can use the information to infer what others are feeling (Lewis et al., 2011). Conscious beings are aware of them, know about their internal states, and are able to predict feelings of others. This type of AI does not yet exist (Hintze, 2016).

AI is functionally linked to Data Sciences (Chen et al., 2012). Data Sciences are conceptual frameworks that deal with Big Data or data sources that are, generally, high-volume (humongous structured and unstructured), high-velocity and high-variety. Data Analytics (DA) is the science of examining raw data with the purpose of drawing conclusions about the inputs. DA involves applying an algorithmic or mechanical process to derive insights as, for instance, running through a number of data sets to look for meaningful correlations between each other. The focus of DA lies in inference (not necessarily statistical) which is the process of deriving conclusions that are solely based on known inputs. Forecasting consumer behaviour, for instance, could be one of those application areas. Data analytics is as such, an interdisciplinary field that uses scientific methods, processes, algorithms and systems to extract knowledge and insights from accessible data delivered in a variety of forms, both structured and unstructured (SAS, 2019a). There are descriptive analytics, diagnostic analytics, predictive analytics and perspective analytics.

\section{The concept of vision}

According to El Namaki (1992: 25): “A vision is a mental perception of the kind of environment an individual, or an organization, aspires to create within a broad time horizon and the underlying conditions for the actualization of this perception". It could also be a "concept for a new and desirable future reality that can be communicated throughout the organization" (ibid: 25). A vision belongs to what we may term a process of direction setting. Direction setting connotes the identification of a vision and a strategy for getting there (Nutt and Backoff, 1997). It requires challenging convocational wisdom and analytically looking for patterns that answer very basic questions about the business we are involved into (El Namaki, 1992).

Individuals perceive their visions and do not ask themselves whether they have one. Having a vision implies involvement, commitment and total immersion (Collins and Porras, 1996). All efforts stem from it and all forces and structures are seen in terms of their relevance to its existence. Issues as rest and reward become secondary as attention is focused on the prime target, i.e. vision fulfilment. A vision has also been realistic and feasible; simple and clear, far but close and derived from a sense of direction (Nutt and Backoff, 1997).

Visions are driven by a need to control an organization's destiny. Contemporary business environments are volatile and carry in them the seeds of disruption (El Namaki, 2018), a process that could lead to the disappearance of a company's competitive advantage overnight. The faster a top management can conceive a future vision where new products and industries are positioned within emerging productmarket constructs, the greater is the ability of the firm to control its destiny and affirm a sense of direction.

International Journal of Management and Applied Research, Vol. 6, No. 1 
A vision is finally formulated by explicitly identifying a domain for competitive behaviour, a set of sources of competitive strength and a profile for resource capability.

\section{How AI is inducing change in business vision formulation and conception?}

Today's business environments are in a state of flux. Powerful forces of Artificial Intelligence and data sciences are inducing far reaching structural shifts in products, processes and industries. These disruptive forces call for compatible visions and congruent conditions of actualization. And they induce fundamental shifts in the building blocks of visions.

\subsection{AI and emerging arenas}

AI technologies will lead to a fundamental restructuring of industries and the emergence of new arenas. An arena is a delineated boundary for combat. Within a business context it would describe a boundary for competitive behaviour among a set of related industries or a segment of the economy. Artificial intelligence will disrupt this segmentation leading to "leapfrog" in the development of new industries, reconfiguring of existing industries and the emergence of new business arenas. Intelligent manufacturing software-defined universal factories will, for example, emerge providing a new industrial arena (Ransbotham, et al., 2017). Emerging future arenas most quoted today include Internet of things IOT, Mobility, Digital logistics, 3D printing, Robotics, Advanced Life Sciences, Cyber security and Big Data.

Business visions will have to reflect this dynamic shift and the ultimate shape of the business environment.

\subsection{AI and competitive advantage}

AI technologies will shift competitive advantage from the material to the intelligent.

AI technologies will reconfigure several dimensions of competition. There is first demand generation. Consumer identification of products and markets will be running through cyber domains including on line outlets and other digital modes. AI will also allow better learning from the past to predict - or change - the future. Intelligence gleaned from historic data would lead to the unravelling of future opportunities and risks. Data-driven analytics will lead to data driven marketing, privileged access to data will become the focus of rivalry among corporations (Ransbotham et al., 2017).

Parameters of competition will undergo fundamental shifts and, with them, the driving forces of business visions.

\subsection{AI and capability construct}

AI technologies will lead to measurable change in the resource profile of the firm.

A vision implies a capability construct. This capability construct is built around three prime components: technology, capital and managerial competence. Artificial intelligence technology-related dimension represents the prime component of this construct. This extend over a wide front that include, among others, Cognitive Computing, Machine Learning, Deep Learning, Predictive APIs, Natural Language Processing, Image Recognition and Speech Recognition. And those will touch some of

International Journal of Management and Applied Research, Vol. 6, No. 1 
the basic foundations of industries from healthcare, marketing, finance and security to logistics and communication.

Capability constructs will constitute a fundamental element of future visions. There is for each vision-induced strategic thrust a capability constructs conducive to that specific vision.

\section{The emerging span of AI derived visions}

Visions within an AI era will be the outcome of a blend of novel arenas, intelligent competition and technology-rooted capabilities. The three forces will interact within a cause and affect pattern. It is more likely than not that AI induced technology will take the lead with novel arenas emerging as a consequence and the capability construct acting as a dependent variable.

Figure 1: The emerging span of AI derived visions

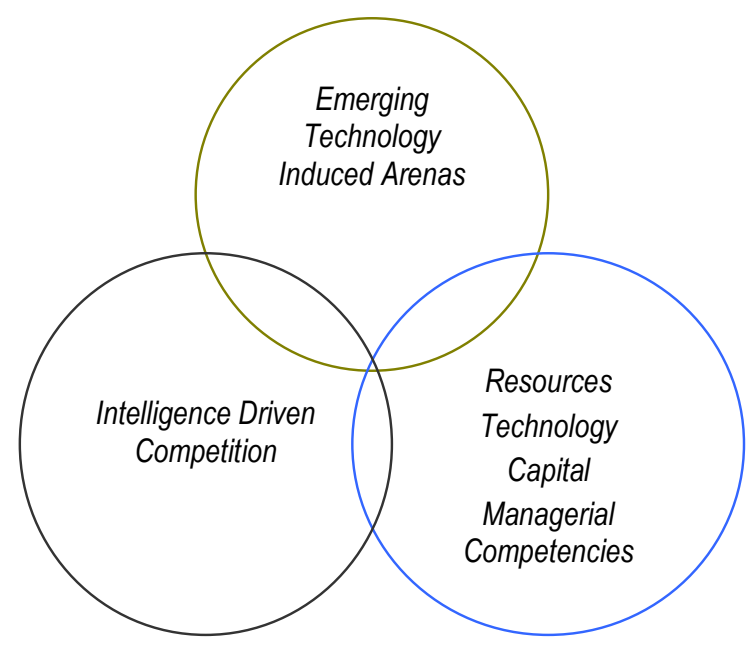

\section{Case evidence}

Vision statements made by several key global corporations reflect the vision shift outlined above. Prime among those is Huawei and Microsoft. New comers include SAS.

\subsection{Huawei}

Huawei's vision stresses the fundamental future change in technologies, markets and management. It states "The fourth industrial revolution - founded on ICT networks and driven by AI - is leading us into an intelligent world where all things will sense, all things will be connected, and all things will be intelligent". Huawei's vision statement also states "All things sensing, more and better connections, bringing everything to the intelligent world". Huawei conceived and expressed the vision within its Global Industry Vision 2025 report. The report outlines future business practices, solutions, and technological innovations in artificial intelligence, 5G, cloud services and the Internet of Things. 


\subsection{Microsoft Corporation}

In 2017, Microsoft released its annual report, where the company added AI into its vision. In reference to the report,

Microsoft is a technology company whose mission is to empower every person and every organization on the planet to achieve more. We strive to create local opportunity, growth, and impact in every country around the world. Our strategy is to build best-in-class platforms and productivity services for an intelligent cloud and an intelligent edge infused with artificial intelligence (Microsoft Corporation, 2017).

Microsoft's corporate vision defines its target market and products. The first component of the vision statement indicates Microsoft's target market, that is, the global market. In addition to selling products to individual customers, the company also sells its products to organizations. The second component of Microsoft's vision statement partly shows what the company strives to achieve: to provide products that can empower customers and assist them with their attainment goals. Additionally, the updated mission statement is strongly aligned with the corporate vision, which is imperative to decision making at corporate level, including setting directions for growing the business and accomplishing its corporate vision.

\subsection{SAS}

SAS, a data analytics organization, conceived a vision that states "To transform a world of data into a world of intelligence" (SAS, 2019b). It envisions a world where better decisions, grounded in trusted data and assisted by the power of analytics are within direct reach. "The goal of cognitive computing is to provide assistance to humans without human assistance". SAS maintains an artificial intelligence portfolio (Hadoop, Visual Text Analytics, SAS Platform, etc.) that enables faster insights and simplifies the end-to-end solution for businesses (SAS, 2019a). It focuses on investment areas supporting continued growth in critical and emerging fields.

\section{Summary and conclusions}

Conceiving a vision has never been as critical as today. The era of data sciences and artificial intelligence is upon us and the disruptive implication of those forces calls for a significant shift in the content of visions and the pertinent visionary leadership.

Artificial Intelligence, a branch of computer science dealing with the simulation of intelligent behaviour, will influence the process of vision conception as well as the ultimate shape of the vision itself. Conventional building stones of visions i.e. arenas, competitive advantage and resources will give way to a new AI induced era of dynamic arenas, intelligent competition and dynamic resource construct.

Cases provided in this article demonstrate the impact of AI on the process of vision conception as well as the outcome of the process. Vision statements formulated within the emerging era by key corporations as Huawei, Microsoft and SAS demonstrate that.

International Journal of Management and Applied Research, Vol. 6, No. 1 


\section{References}

1. Chen, H.; Chiang, R.H.L. and Storey, V.C. (2012), "Business intelligence and analytics: From big data to big impact", MIS quarterly, Vol. 36 No. 4, pp. 1165-1188.

2. Collins, J. C. and Porras, J. I. (1996), "Building Your Company's Vision”, Harvard Business Review, Vol. 74, No. 5, pp. 65-78

3. El-Namaki, M. S. S. (1992), "Creating a Corporate Vision", Long Range Planning, Vol. 25, No. 6, pp. 25-29. https://doi.org/10.1016/0024-6301(92)90166-Y

4. El Namaki, M. S. S. (2017), "Disruption and the Changing Concept of Leadership", International Journal of Management and Applied Research, Vol. 4, No. 2, pp. 122129. https://doi.org/10.18646/2056.42.17-010

5. El Namaki, M. S. S. (2018), "Disruption in Business Environments: A Framework and Case Evidence", International Journal of Management and Applied Research, Vol. 5, No. 1, pp. 1-7. https://doi.org/10.18646/2056.51.18-001

6. Friedlander, D. And Franklin, S. (2008), "LIDA and a theory of mind", in: Wang, P.; Goertzel, B. and Franklin S. (Eds.), in: Artificial General Intelligence 2008: Proceedings of the First AGI Conference, The Netherlands: IOS Press.

7. Hintze, A. (2016), "Understanding the four types of AI, from reactive robots to selfaware beings", The Conversation, [Online] Available from: http://theconversation.com/understanding-the-four-types-of-ai-from-reactive-robots-toself-aware-beings-67616 [Accessed on 1 March 2019].

8. Huawei (2018), Global Industry Vision 2025, [Online] Available from: https://www.huawei.com/minisite/giv/en/era.html [Accessed on 2 March 2019].

9. Lewis, P. R.; Chandra, A.; Parsons, S.; Robinson, E.; Glette, K.; Bahsoon, R.; Torresen, J. and Yao, X. (2011), "A Survey of Self-Awareness and Its Application in Computing Systems", in: 2011 Fifth IEEE Conference on Self-Adaptive and SelfOrganizing Systems Workshops, https://doi.org/10.1109/SASOW.2011.25

10. Microsoft Corporation (2017), Annual Report Pursuant to Section 13 or 15(d) of the Securities Exchange Act of 1934 For the Fiscal Year Ended June 30, 2017 [Online] Available from: https://www.sec.gov/Archives/edgar/data/789019/000156459017014900/msft10k_20170630.htm [Accessed on 1 March 2019].

11. Nutt, P. C. and Backoff, R. W. (1997), “Crafting Vision”, Journal of Management Inquiry, Vol. 6, No. 4, pp. 308-328. https://doi.org/10.1177/105649269764007

12. Ransbotham, S.; Kiron, D.; Gerbert, P.; and Reeves, M. (2017), "Reshaping Business With Artificial Intelligence", MIT Sloan Management Review, Vol. 59, No. 1, pp. 117.

13. SAS (2019a), Big data analytics: what it is and why it matters [Online] Available from: https://www.sas.com/en gb/insights/analytics/big-data-analytics.html [Accessed on 12 January 2019].

14. SAS (2019b), Vision and Mission [Online] Available from: https://www.sas.com/en_gb/company-information/vision-mission.html [Accessed on 12 January 2019].

International Journal of Management and Applied Research, Vol. 6, No. 1 